\title{
Analytical Solution for Electrical Problem Forced by a Finite-Length Needle Electrode: Implications in Electrostimulation
}

\author{
Ricardo Romero-Méndez $\mathbb{D}^{1},{ }^{1}$ Francisco G. Pérez-Gutiérrez, ${ }^{1}$ \\ Francisco Oviedo-Tolentino, ${ }^{1}$ and Enrique Berjano $\mathbb{D}^{2}$ \\ ${ }^{1}$ Facultad de Ingeniería, Universidad Autónoma de San Luis Potosí, San Luis Potosí, SLP 78290, Mexico \\ ${ }^{2}$ BioMIT, Department of Electronic Engineering, Universitat Politècnica de València, Camino de Vera, \\ Valencia, 46022, Spain \\ Correspondence should be addressed to Ricardo Romero-Méndez; rromerom@uaslp.mx
}

Received 8 August 2018; Accepted 2 May 2019; Published 19 May 2019

Academic Editor: Xuping Zhang

Copyright ( 2019 Ricardo Romero-Méndez et al. This is an open access article distributed under the Creative Commons Attribution License, which permits unrestricted use, distribution, and reproduction in any medium, provided the original work is properly cited.

\begin{abstract}
Needle electrodes, widely used in clinical procedures, are responsible for creating an electric field in the treated biological tissue. This is achieved by setting a constant voltage along the length of their metallic section. In accordance with Laplace's equation, the electric field is spatially non-uniform around the electrode surface. Mathematical modelling can provide useful information on the spatial distribution of electrical fields. Indeed, exact solutions for the electrical problem are indispensable for validating numerical codes. All the analytical models developed to date to solve the needle electrode electrical problem have been one-dimensional models, which assumed an electrode of infinite length. We here propose the first analytical solution based on a two-dimensional model that considers the real length of the electrode in which the Laplace equation is solved through the method of separation of variables, dealing with the nonhomogeneous source term and boundary conditions by Green's functions. On assuming a needle electrode of given length, the problem combines boundary conditions on the electrode boundary (of the first and second kind). Since this rules out using the Sturm-Liouville Theorem, the problem is decomposed into two different problems and the principle of superposition is used. The solution obtained can reproduce a reasonable electric field around the electrode, especially the edge effect characterized by an extremely high gradient around the electrode tip.
\end{abstract}

\section{Introduction}

Needle electrodes consist of a thin cylindrical metal piece partially coated with plastic. The electrode is really the exposed part (i.e., the non-insulated portion, also known as the conductive tip) beveled to a sharp point. During clinical use, the exposed part is completely surrounded by tissue. Single shaft needle electrodes are employed in many clinical procedures that require the creation of an electric field and the resulting current density field. To create this field a voltage difference has to be set between two electrodes, one of which is the needle electrode and the other is a large area electrode, also known as a patch, reference electrode, or dispersive electrode. A large variety of electrodes for soft tissue have been developed, such as the plain, single internally cooled, cluster internally cooled, expandable and wet electrodes [1]. The target tissue is the region near the needle because the current density there is high, producing the desired effect over the tissue. The accurate modeling of the electrical problem of current circulation between needle electrode and dispersive electrode through the tissue is important to determine if the desired effect can be obtained. Many clinical procedures employ needle electrodes of several types, such as electrical stimulation of excitable tissues $[2,3]$, characterization of tissue impedance [4], and thermal destruction of tissue by applying radiofrequency $(\mathrm{RF})$ current $[5,6]$.

Mathematical modeling is able to provide useful information on the electrical behavior of needle electrodes, 


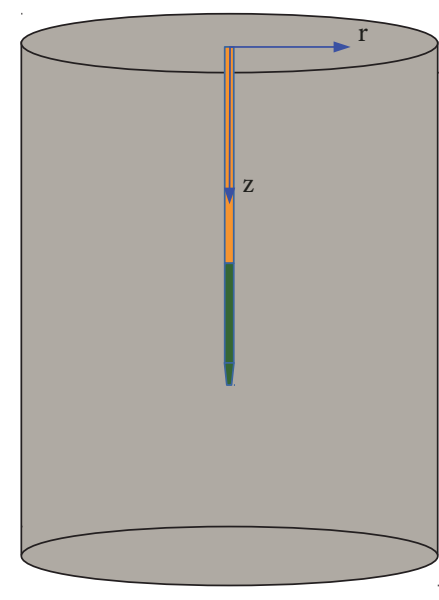

(a)

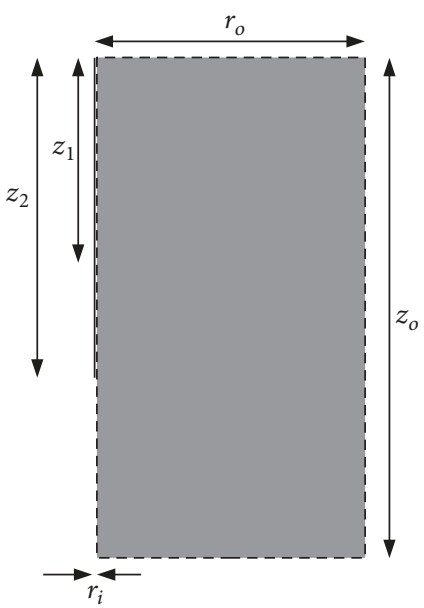

(b)

Figure 1: (a) The model represented a needle electrode comprised of a conductive tip (green zone) and a portion coated with a plastic material (orange zone). The electrode is completely surrounded by tissue (gray zone). (b) The two-dimensional model presents axial symmetry around the electrode axis, and its domain is limited by the electrode surface (radius $r_{i}$ ), tissue dimension ( $\operatorname{radius} r_{o}$ ), and height $z_{o}$. The needle electrode is modeled by a boundary zone of length $z_{2}-z_{1}$.

especially on the spatial distribution of the electrical field $\mathbf{E}$ and current density $\mathbf{J}$ produced in the tissue next to the electrode. The general electric behavior of any electrode is basically as follows: a voltage $V$ is uniformly set along the entire surface of the electrode, while both current density $\mathbf{J}$ and electric field $\mathbf{E}$ distributions are spatially non-uniform at the electrode surface, as they result from solving the Laplace equation (assuming electrical conductivity $\sigma$ to be constant). This behavior has previously been correctly reproduced by mathematical models using numerical methods [7-10]. Since the numerical simulation has to be able to accurately reproduce the physical situation expressed by the partial differential equations that model the process, comparing their results with any existing benchmark analytical solutions would be one way of validating the numerical simulations. In other words, exact solutions for the electrical problem are indispensable to validate numerical codes, since the mesh refinements used in the codes should be able to capture the large gradients of voltage and electric field around the needle electrode, which the analytical solution must accurately determine. Closed form solutions may also be useful in designing control strategies [11], as well as for a quick check of operating conditions in clinical procedures.

To our knowledge, all the previous analytical models that aimed to solve the electrical problem produced by a needle electrode were one-dimensional models and assumed an electrode of infinite length [12-14]. Since the real length of the electrode (non-insulated tip) was not considered, their solutions were not able to reproduce the edge effect characterized by an extremely high gradient around the tip and the joint between the insulated and non-insulated portions $[9,10]$. Our goal was thus to find a two-dimensional analytical solution for the electric fields created by a needle electrode of a given length.

\section{Materials and Methods}

2.1. Description of the Model Geometry. The monopolar needle-like electrode is a small radius cylindrical device, which is inserted into a tissue. A voltage is applied between the electrode's surface and a grounded patch located on the skin of the patient so that the current circulates through the tissue. The spatial distribution of voltage and current throughout all the tissues that comprise the electrical circuit is really very complex, but since the voltage decays rapidly as the radial distance from the electrode is increased, it is standard to model the electrical problem of monopolar cylindrical electrodes using a cylindrical coordinate system, by looking for planes or symmetry axes. This allows to model a three-dimensional physical problem with a simplified two-dimensional geometry (axisymmetric case) [15].

The analytical model represents a physical situation in which a needle electrode with radius $r_{i}$ is completely surrounded by homogeneous tissue (see Figure 1(a)). The reference electrode is assumed to be placed far from the needle electrode and has a comparatively much larger area. As the electrode radius is much smaller than the tissue, we assume the domain comprises an annular section of tissue of height $z_{0}=150 \mathrm{~mm}$ with inner radius $r_{i}=0.75 \mathrm{~mm}$ and outer radius $r_{0}=60 \mathrm{~mm}$ (see Figure 1(b)). Since the conductive portion of the needle electrode is assumed to be in the middle of the tissue block, its length is $z_{2}-z_{1}$.

The boundary $r=r_{i}$ is divided into three sections in order to model the finite length of the electrode: $0 \leq z \leq z_{1}$ is the insulated portion of the electrode, $z_{1}<z<z_{2}$ is the conductive portion of the electrode, i.e., through which the electrical current flows, and finally $z \geq z_{2}$ is the tissue below the electrode. The boundary conditions are insulation on the boundary $r=r_{i}$, except for the conductive portion of 
the electrode, where we assume a uniform voltage $V_{0}$. The top axially normal surface $(z=0)$ is considered electrically insulated, while both outer radius boundary $\left(r=r_{0}\right)$ and the bottom surface $\left(z=z_{0}\right)$ are held at zero voltage, mimicking the reference electrode.

The electrical problem is solved by the Laplace Equation

$$
\nabla \cdot(\sigma \nabla \mathrm{V})=0
$$

where $\sigma$ is the electrical conductivity of tissue $(\mathrm{S} / \mathrm{m})$, considered constant in the model, and $V$ is the electric voltage $(\mathrm{V})$. The electric field $\mathbf{E}$ and electric current density $\mathbf{J}$ are obtained from

$$
\begin{aligned}
& \mathbf{E}=-\nabla \mathbf{V} \\
& \mathbf{J}=\sigma \mathbf{E}
\end{aligned}
$$

Equation (1) is a quasi-steady simplification of the basic relationship between electric current density and charge, called continuity equation, which is obtained by manipulation of Maxwell's equations. The continuity equation states that the net electrical current flowing in and out of a volume is equal to the rate of change of electrical charge inside the volume. Since the time scale of the electrical phenomenon is much shorter than that of other associated phenomena, such as thermal damage or electrostimulation, we can simplify in this case the continuity equation as Laplace equation (1). On the other hand the quasi-static approximation is suitable due to the low value of the frequencies, which implies that the wavelength is much larger than the physical dimensions, and hence no electromagnetic wave is present. In practical terms this means that the electrical problem is mathematically solved as if it was a DC problem, and not a problem with AC signals.

In practical terms, the electrical problem is analytically solved through the method of separation of variables, where the nonhomogeneous boundary conditions are handled using Green's functions.

2.2. Calculation (Analytical Solution). Considering that in this problem the voltage $V$ is assumed to be a function of the radial $(r)$ and axial coordinates $(z)$, Equation (1) can be stated as

$$
\frac{1}{r} \frac{\partial}{\partial r}\left(r \frac{\partial V}{\partial r}\right)+\frac{\partial^{2} V}{\partial^{2} z}=0
$$

subject to the following boundary conditions:

$$
\begin{aligned}
{\left[\frac{\partial V}{\partial z}\right]_{(r, 0)} } & =0 \\
V\left(r, z_{0}\right) & =0 \\
V\left(r_{0}, z\right) & =0
\end{aligned}
$$

$$
\begin{aligned}
& {\left[\frac{\partial V}{\partial r}\right]_{\left(r_{i}, z\right)}=0 \quad \text { if } z<z_{1}} \\
& V\left(r_{i}, z\right)=V_{0} \quad \text { if } z_{1} \leq z \leq z_{2} \\
& {\left[\frac{\partial V}{\partial r}\right]_{\left(r_{i}, z\right)}=0 \quad \text { if } z>z_{2}}
\end{aligned}
$$

where $V_{0}$ is the voltage applied to the conductive portion of the needle electrode. The electrical conductivity of the electrode is very large compared to that of the tissue, and for that reason the active surface of the electrode can be considered at a constant voltage. Moreover, since the metallic electrode is not considered as part of the domain, the solution does not depend on the electrical properties of the electrode.

As the electrode radius is much smaller than the radial dimension of the mathematical domain, we considered the region $r=r_{\mathrm{i}}$ and $z>z_{2}$ as a quasi-symmetric region. A symmetric condition is mathematically equivalent to an insulated boundary.

Since this is a problem with combined boundary conditions (of the first and second kind) on the boundary $r=r_{i}$, it is not suitable for direct solution by the Sturm-Liouville Theorem. The option is to decompose it into two different problems and use the principle of superposition.

$$
V(r, z)=V_{1}(r, z)+V_{2}(r, z)
$$

The decomposition of $V$ into $V_{1}$ and $V_{2}$ was used to make it possible to solve a mathematical problem, which combines both Dirichlet and Neumann boundary conditions at $r=r_{\mathrm{i}}$. This decomposition is possible because of the linearity of the governing equation and boundary conditions, which allows the use of the principle of superposition.

$V_{1}$ is obtained solving

$$
\frac{1}{r} \frac{\partial}{\partial r}\left(r \frac{\partial V_{1}}{\partial r}\right)+\frac{\partial^{2} V_{1}}{\partial^{2} z}=0
$$

subject to the following boundary conditions:

$$
\begin{aligned}
{\left[\frac{\partial V_{1}}{\partial z}\right]_{(r, 0)} } & =0 \\
V_{1}\left(r, z_{0}\right) & =0 \\
V_{1}\left(r_{0}, z\right) & =0 \\
V_{1}\left(r_{i}, z\right) & =V_{T} \quad \text { if } z<z_{1} \\
V_{1}\left(r_{i}, z\right) & =V_{0} \quad \text { if } z_{1} \leq \mathrm{z} \leq z_{2} \\
V_{1}\left(r_{i}, z\right) & =V_{T} \quad \text { if } z>z_{2}
\end{aligned}
$$

where $V_{T}$ is a constant voltage with no physical significance (i.e., it does not correspond to the real applied voltage $V_{0}$ ), but was chosen to guarantee the final satisfaction of the original problem expressed by Equations (4)-(10). Details of how to 
choose the value of $V_{T}$ are given in Section 4. By using the method of separation of variables, the solution to Equations (12)-(18) can be written as

$$
\begin{aligned}
& V_{1}(r, z)=\sum_{m=1}^{\infty} A_{m} \cos \left(\eta_{m} z\right) \\
& \cdot\left[K_{0}\left(\eta_{m} r\right)-\frac{K_{0}\left(\eta_{m} r_{0}\right)}{I_{0}\left(\eta_{m} r_{0}\right)} I_{0}\left(\eta_{m} r\right)\right]
\end{aligned}
$$

where $I_{0}\left(\eta_{m} r\right)$ and $K_{0}\left(\eta_{m} r\right)$ are the modified Bessel functions of the first and second kind, respectively, and $\eta_{m}$ are the eigenvalues for this problem

$$
\eta_{m}=\frac{(2 m-1) \pi}{2 z_{0}}
$$

and

$$
A_{m}=\frac{2 I_{0}\left(\eta_{m} r_{0}\right)}{z_{0} \eta_{m}} \frac{\left[V_{0}\left(\sin \left(\eta_{m} z_{2}\right)-\sin \left(\eta_{m} z_{1}\right)\right)+V_{T}\left(\sin \left(\eta_{m} z_{1}\right)+\sin \left(\eta_{m} z_{0}\right)-\sin \left(\eta_{m} z_{2}\right)\right)\right]}{K_{0}\left(\eta_{m} r_{i}\right) I_{0}\left(\eta_{m} r_{0}\right)-K_{0}\left(\eta_{m} r_{0}\right) I_{0}\left(\eta_{m} r_{i}\right)}
$$

$V_{2}$ is obtained solving

$$
\frac{1}{r} \frac{\partial}{\partial r}\left(r \frac{\partial V_{2}}{\partial r}\right)+\frac{\partial^{2} V_{2}}{\partial^{2} z}=0
$$

subject to the following boundary conditions:

$$
\begin{aligned}
& {\left[\frac{\partial V_{2}}{\partial z}\right]_{(r, 0)}=0} \\
& V_{2}\left(r, z_{0}\right)=0 \\
& V_{2}\left(r_{0}, z\right)=0 \\
& {\left[\frac{\partial V_{2}}{\partial z}\right]_{r_{i}}=-\left[\frac{\partial V_{1}}{\partial z}\right]_{r_{i}} \quad \text { if } z<z_{1}} \\
& {\left[\frac{\partial V_{2}}{\partial z}\right]_{r_{i}}=0 \quad \text { if } z_{1} \leq \mathrm{z} \leq z_{2}} \\
& {\left[\frac{\partial V_{2}}{\partial z}\right]_{r_{i}}=-\left[\frac{\partial V_{1}}{\partial z}\right]_{r_{i}} \quad \text { if } z>z_{2}}
\end{aligned}
$$

Boundary conditions (27) and (28) guarantee that, by superposing the solutions for $V_{1}$ and $V_{2}$, the gradients $\partial V / \partial z$ at $r_{i}$ are zero for sections $0 \leq \mathrm{z} \leq z_{1}$ and $z_{2} \leq \mathrm{z} \leq z_{0}$. The solution to Equations (22) to (28) is

$$
\begin{aligned}
& V_{2}(r, z) \\
& \quad=\sum_{n=1}^{\infty} C_{n} \cos \left(\eta_{n} z\right)\left[K_{0}\left(\eta_{n} r\right)-\frac{K_{0}\left(\eta_{n} r_{0}\right)}{I_{0}\left(\eta_{n} r_{0}\right)} I_{0}\left(\eta_{n} r\right)\right]
\end{aligned}
$$

where

$$
\begin{aligned}
C_{n} & =\frac{-2 I_{0}\left(\eta_{n} r_{0}\right)}{z_{0} \eta_{n}} \\
& . \frac{\sum_{m=1}^{\infty} A_{m} \eta_{m}\left[K_{1}\left(\eta_{m} r_{i}\right)+K_{0}\left(\eta_{m} r_{0}\right) I_{1}\left(\eta_{m} r_{i}\right) / I_{0}\left(\eta_{m} r_{0}\right)\right] \Psi}{K_{1}\left(\eta_{n} r_{i}\right) I_{0}\left(\eta_{n} r_{0}\right)+K_{0}\left(\eta_{n} r_{0}\right) I_{1}\left(\eta_{n} r_{i}\right)}
\end{aligned}
$$

and

$$
\begin{aligned}
\Psi= & {\left[\frac{\sin \left(\left(\eta_{m}+\eta_{n}\right) z_{1}\right)}{2\left(\eta_{m}+\eta_{n}\right)}+\frac{\sin \left(\left(\eta_{m}-\eta_{n}\right) z_{1}\right)}{2\left(\eta_{m}-\eta_{n}\right)}\right] } \\
& +\left[\frac{\sin \left(\left(\eta_{m}+\eta_{n}\right) z\right)}{2\left(\eta_{m}+\eta_{n}\right)}+\frac{\sin \left(\left(\eta_{m}-\eta_{n}\right) z\right)}{2\left(\eta_{m}-\eta_{n}\right)}\right]_{z_{2}}^{z_{0}}
\end{aligned}
$$

In the special case $\eta_{n}=\eta_{m}$

$$
\frac{\sin \left(\left(\eta_{m}-\eta_{n}\right) z\right)}{2\left(\eta_{m}-\eta_{n}\right)} \rightarrow \frac{z}{2}
$$

Once both $V_{1}$ and $V_{2}$ are calculated and superposed to obtain $V$, the magnitude of the electric field vector $\mathbf{E}$ is calculated as

$$
|\mathbf{E}|=\sqrt{\left(\frac{\partial V}{\partial r}\right)^{2}+\left(\frac{\partial V}{\partial z}\right)^{2}}
$$

while the magnitude of the current density vector $\mathbf{J}$ is calculated as

$$
|\mathbf{J}|=\sigma \sqrt{\left(\frac{\partial V}{\partial r}\right)^{2}+\left(\frac{\partial V}{\partial z}\right)^{2}}
$$

After solving the equations analytically, Matlab (Mathworks, Natick, MA, USA) was used to compute each value at any particular point, so that all the plots were created by choosing a particular set of points.

\section{Results}

As mentioned, the value of $V_{T}$ must be suitably chosen to achieve a mathematical result compatible with the physics, i.e., a uniform voltage distribution along the electrode surface. Figure 2 shows the voltage distributions obtained for three different values of $V_{T}$.

We found that if a small value is chosen for $V_{T}$ $\left(V_{T}=0.1 \times V_{0}\right)$, the resulting voltage $V$ along the electrode surface is not constant and shows a convex shape, with higher voltage at both ends (see Figure 2(a)). In contrast, if a large value is chosen $\left(V_{T}=0.8 \times V_{0}\right)$, the resulting voltage $V$ along the 


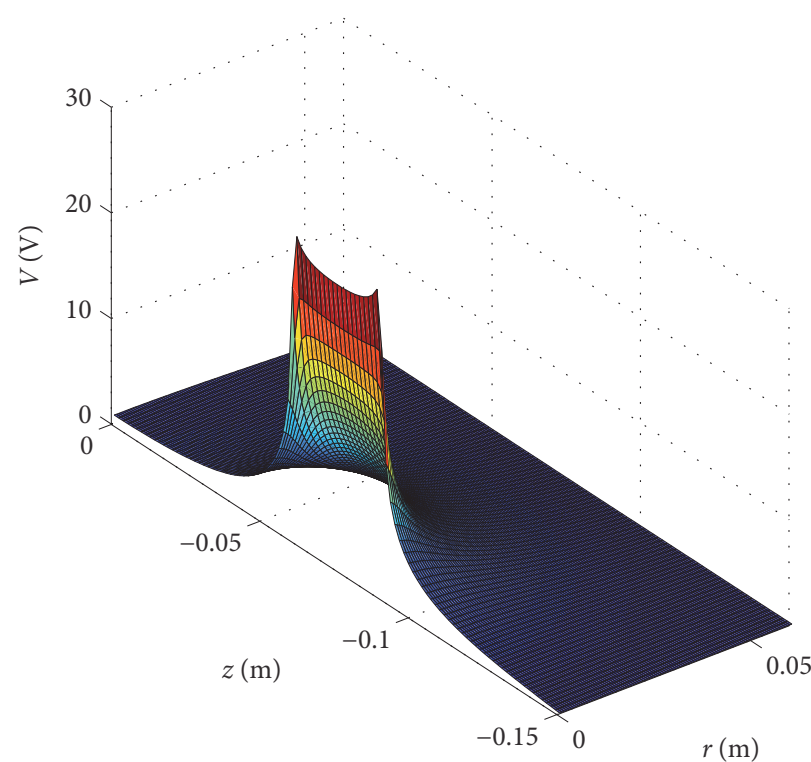

(a)

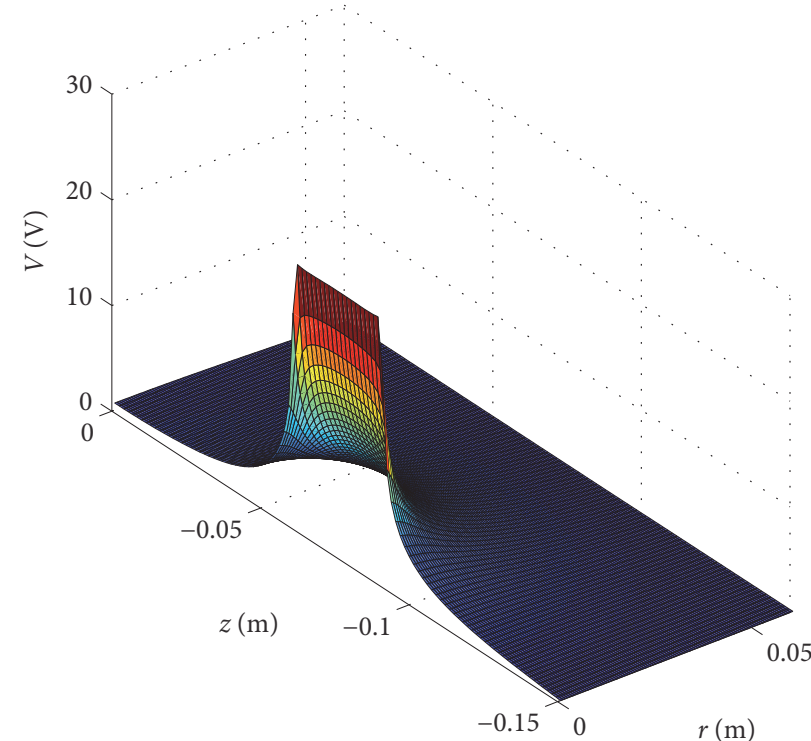

(b)

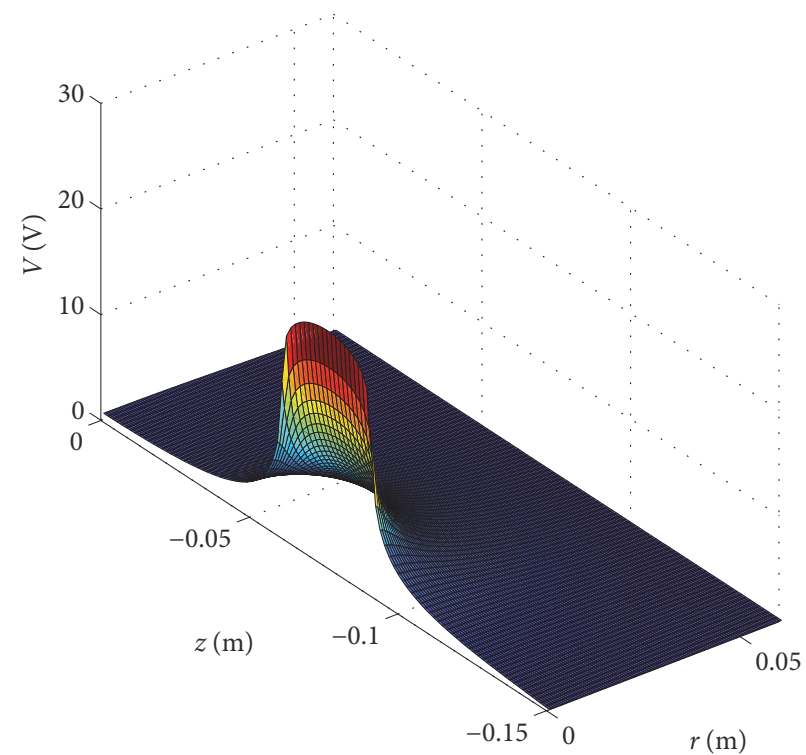

(c)

Figure 2: Voltage distributions computed for different values of $V_{\mathrm{T}}$. (a) $V_{\mathrm{T}}=0.1 \times V_{0}$; (b) $V_{\mathrm{T}}=0.378 \times V_{0}$; and (c) $V_{\mathrm{T}}=0.8 \times V_{0}$. $V_{0}$ is the applied voltage of value $(25 \mathrm{~V})$. Plots are of a needle electrode of $1.5 \mathrm{~mm}$ diameter $\left(r_{i}=0.75 \mathrm{~mm}\right)$ and conductive portion of $30 \mathrm{~mm}$. The following domain dimensions were considered: $r_{0}=60 \mathrm{~mm}, z_{1}=60 \mathrm{~mm}, z_{2}=90 \mathrm{~mm}$, and $z_{0}=150 \mathrm{~mm}$.

electrode surface is not constant and shows a concave shape, with lower voltage at both ends (see Figure 2(c)). In the case of a needle electrode with $1.5 \mathrm{~mm}$ diameter $\left(r_{i}=0.75 \mathrm{~mm}\right)$ and conductive portion of $30 \mathrm{~mm}$, we found that a value $V_{T}$ $=0.378 \times V_{0}$ produced a constant voltage along the electrode surface, which makes sense from a physical point of view (see Figure 2(b)). The effect of changing $V_{T}$ is clearer in Figure 3, which shows the voltage profile on the electrode surface $(r=$ $\left.r_{i}\right)$.

However, the value of $V_{T}$ depends on the electrode dimensions. For instance, when the electrode diameter is
$1 \mathrm{~mm}\left(r_{i}=0.5 \mathrm{~mm}\right) V_{T}$ has to be $0.335 \times V_{0}$, while that for $2 \mathrm{~mm}$ $\left(r_{i}=1 \mathrm{~mm}\right) V_{T}$ has to be $0.435 \times V_{0}$. Figure 4 shows voltage plots for these electrode diameter values. Note that these values of $V_{T}$ produce a uniform voltage along the electrode surface.

Similarly, different values of $V_{\mathrm{T}}$ have to be found for different lengths of the conductive portion of the electrode. Importantly, in the search for the suitable $V_{\mathrm{T}}$ value, the dimensions of the domain must be sufficiently large in the $z$ direction; otherwise it is difficult to find a value $V_{\mathrm{T}}$ that meets the requirement. Figure 5 shows the voltage 


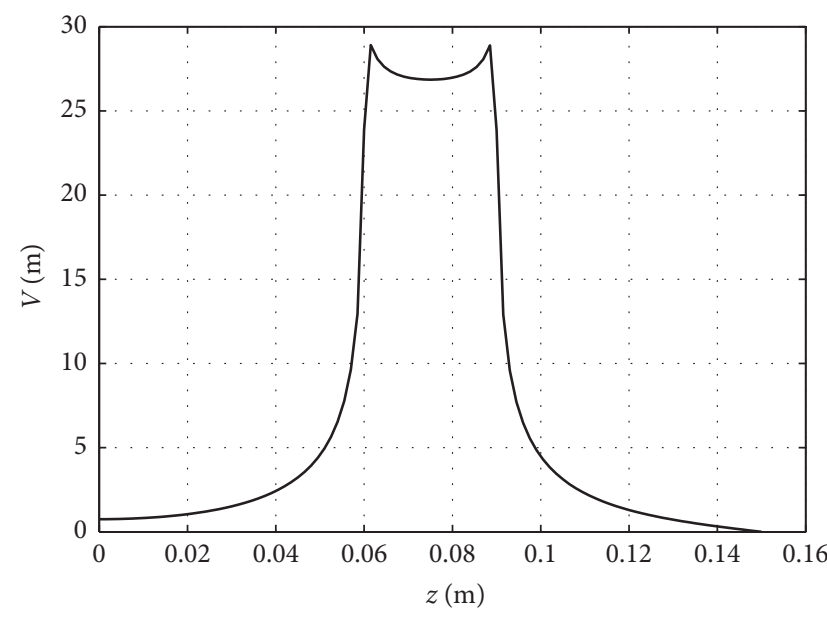

(a)

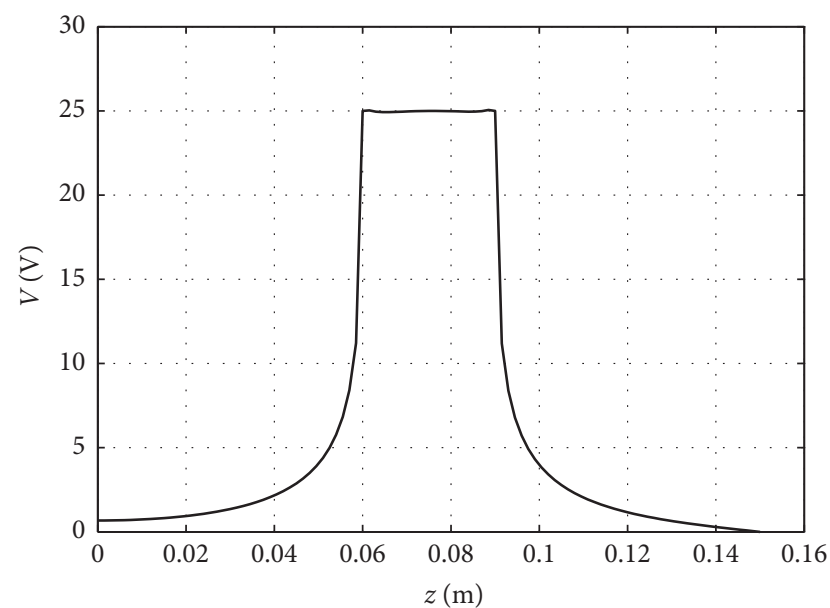

(b)

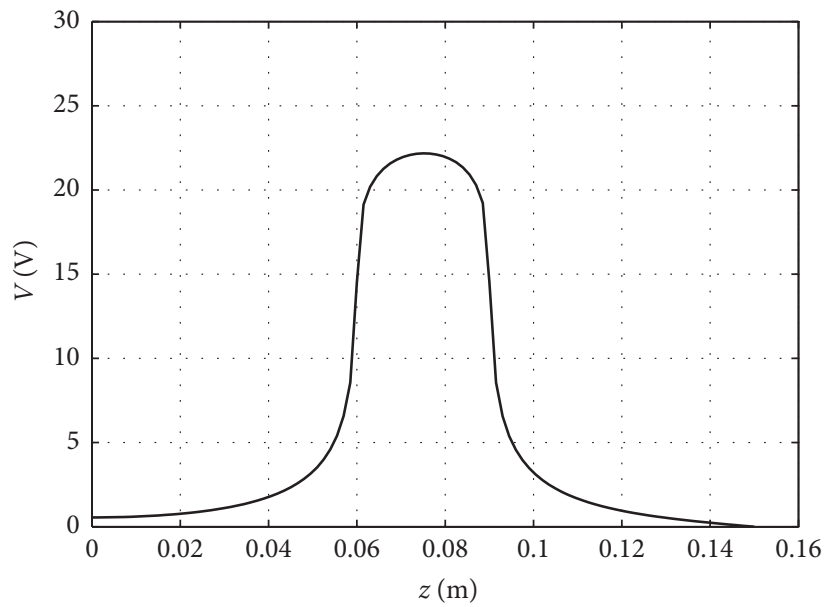

(c)

Figure 3: Voltage profiles at $r=r_{i}$ computed for different values of $V_{T}$. (a) $V_{\mathrm{T}}=0.1 \times V_{0}$; (b) $V_{\mathrm{T}}=0.378 \times V_{0}$; and (c) $V_{\mathrm{T}}=0.8 \times V_{0}$. $V_{0}$ is the applied voltage of value $(25 \mathrm{~V})$. Plots are of a needle electrode of $1.5 \mathrm{~mm}$ diameter $\left(r_{i}=0.75 \mathrm{~mm}\right)$ and conductive portion of $30 \mathrm{~mm}$. The following domain dimensions were considered: $r_{0}=60 \mathrm{~mm}, z_{1}=60 \mathrm{~mm}, z_{2}=90 \mathrm{~mm}$, and $z_{0}=150 \mathrm{~mm}$.

distribution obtained for two different values of the length of the conductive portion. Note that the domain had to be enlarged for longer lengths.

Once $V$ had been obtained, the magnitude of the vectors $\mathbf{E}$ (electric field) and $\mathbf{J}$ (current density) were calculated from the distribution of $\partial V / \partial r$ and $\partial V / \partial z$ as expressed in Eqs. (33) and (34). For instance, Figure 6 shows the profile distribution $\partial V / \partial r$ computed on the symmetry axis. Note that the value of $V_{T}$ was suitably changed to achieve a uniform voltage on the electrode surface.

The combination of these two terms made it possible to plot the vector field. For instance, Figure 7 shows the vector distributions of the current density $(\mathrm{J})$, in which the edge effect, characterized by an extremely high value of $\mathbf{J}$ at the electrode edges, is easily appreciated. This behavior is even more marked when the Joule heating $(q)$ is plotted. This term is the volumetric power density $\left(\mathrm{W} / \mathrm{m}^{3}\right)$ in the tissue caused by the electrical current flow and is given by $q=$ $\mathbf{J} \cdot \mathbf{E}$. In some clinical applications, such as radiofrequency ablation, this term is responsible for creating a thermal lesion. Figure 8 shows an example of this distribution. It can be seen that higher power is applied at the electrode tip than at the proximal edge (where the insulated and exposed parts meet).

\section{Discussion}

In this study we found a novel analytical solution for the twodimensional electrical problem associated with a limitedlength needle electrode. To date, all the analytical solutions have been based on an oversimplified scenario in which the electrode was assumed to have an infinite length [1214] and did not reflect the characteristic performance of the electric field around the electrode, especially at the tips.

While the solution was obtained by using standard mathematical methods (separation of variables), an original approach based on the principle of superposition was used to 


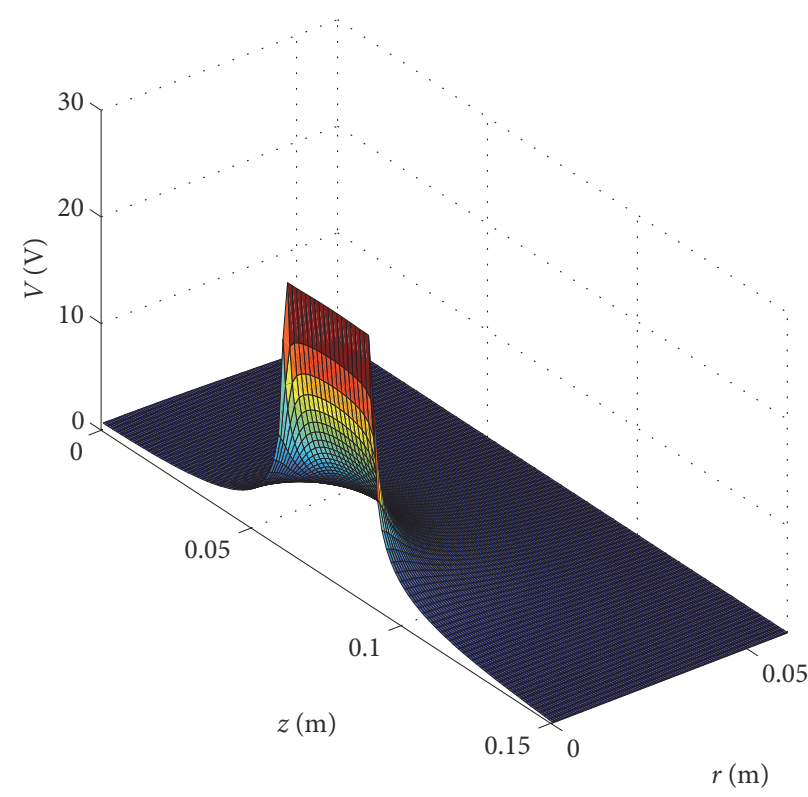

(a)

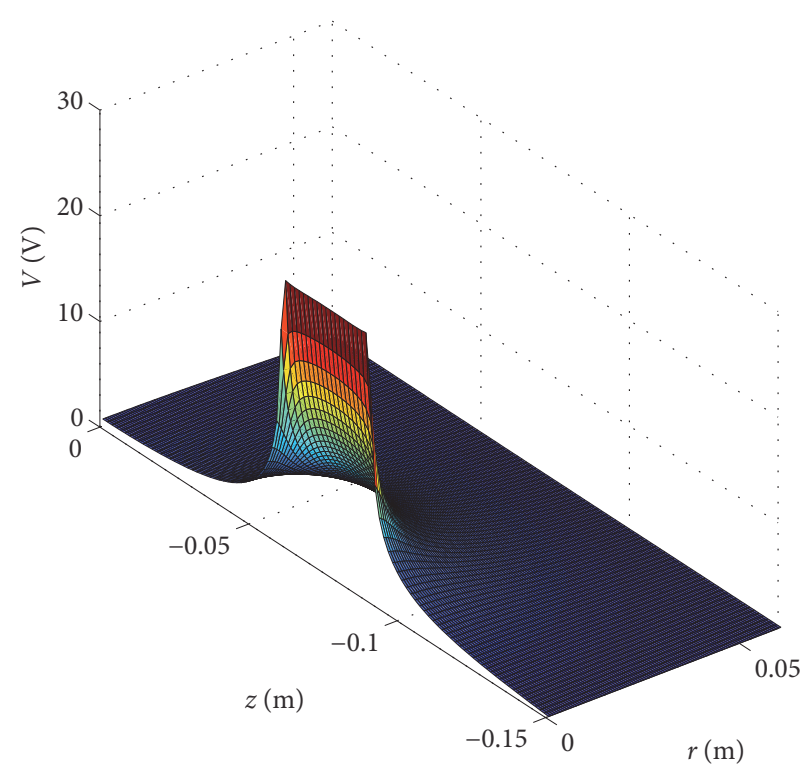

(b)

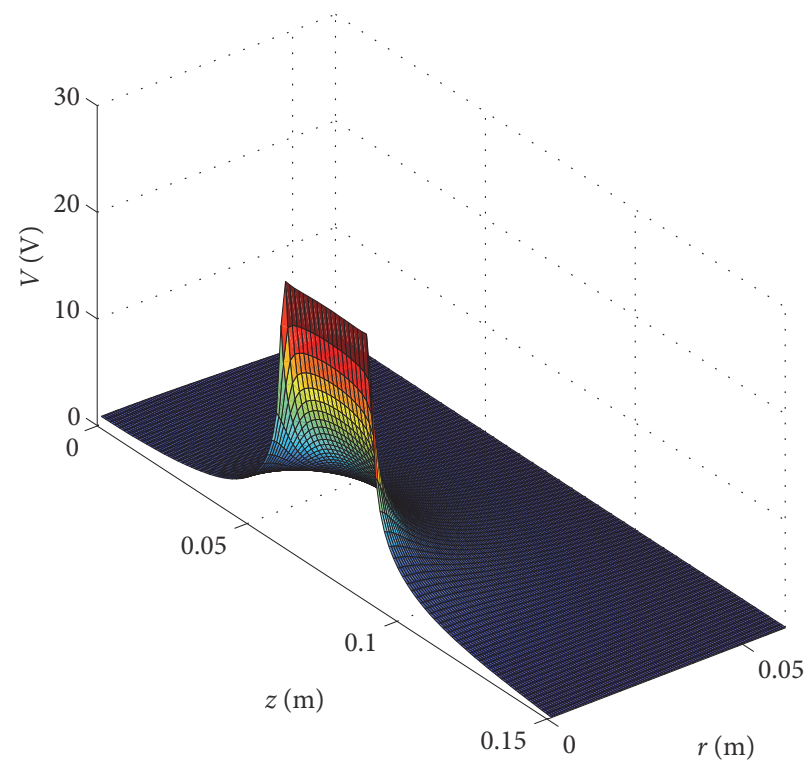

(c)

FIGURE 4: Voltage distributions computed for three different values of electrode diameter. In each case the value of $V_{T}$ was suitably changed to achieve a uniform voltage on the electrode surface. (a) $1 \mathrm{~mm}$ diameter $\left(r_{i}=0.5 \mathrm{~mm}\right)$ with $V_{\mathrm{T}}=0.335 \times V_{0}$; (b) $1.5 \mathrm{~mm}$ diameter $\left(r_{i}=0.75 \mathrm{~mm}\right)$ with $V_{\mathrm{T}}=0.378 \times V_{0}$; and (c) $2 \mathrm{~mm}$ diameter $\left(r_{i}=1 \mathrm{~mm}\right)$ with $V_{\mathrm{T}}=0.435 \times V_{0} . V_{0}$ is the applied voltage of value $(25 \mathrm{~V})$. Plots are of a needle electrode with a conductive portion of $30 \mathrm{~mm}$. The following domain dimensions were considered: $r_{0}=60 \mathrm{~mm}, z_{1}=60 \mathrm{~mm}, z_{2}=90 \mathrm{~mm}$, and $z_{0}=150 \mathrm{~mm}$.

handle the combined boundary conditions on the electrodetissue boundary. The solution obtained has the minor problem of requiring the search for an appropriate value for the parameter $V_{T}$ in order to achieve a uniform voltage on the electrode surface, which is the only thing that makes sense from a physical point of view. In the future this search could be automated for specific electrode dimensions. For now, the proposed solution realistically represents the electric behavior of a needle electrode and provides a reliable and useful analytic solution that can be used by researchers to test their own codes before running extensive numerical experiments, in designing control strategies, as well as for a quick check of the operating conditions in clinical procedures. Moreover, even though the analytical solution is contextualized in the medical field of needle electrodes, it could also be useful for other scientific and industrial applications in which a voltage is applied between a thin metal cylinder and a remote reference electrode. 


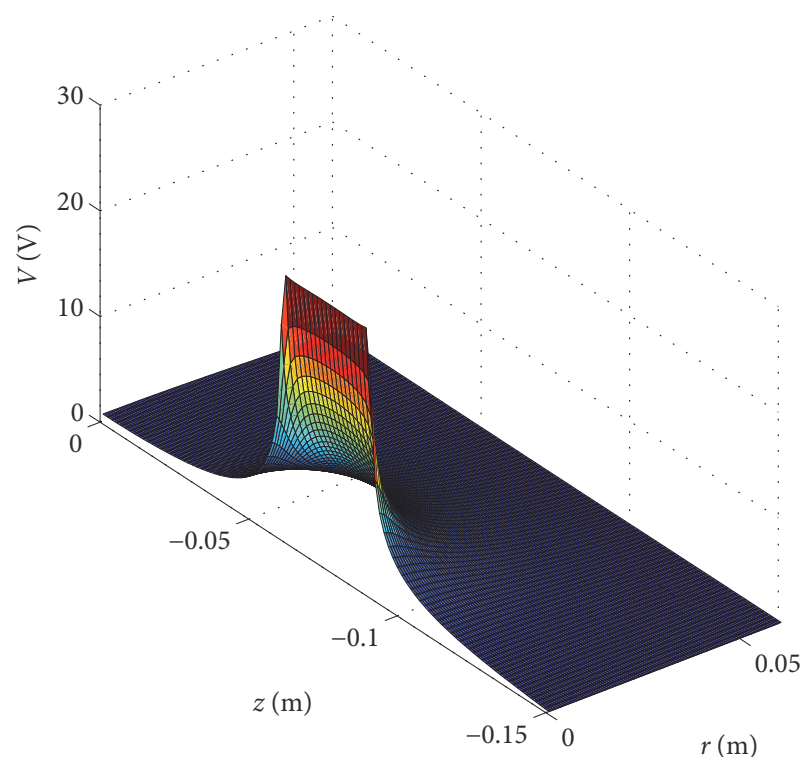

(a)

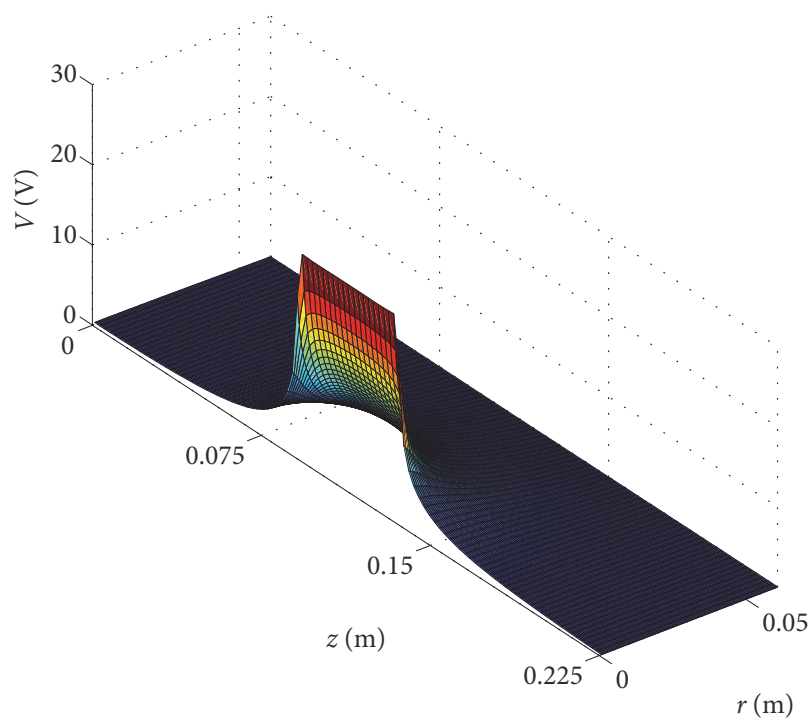

(b)

FIGURE 5: Voltage distributions computed for two different lengths of conductive portion. In each case the value of $V_{T}$ was suitably changed to achieve a uniform voltage on the electrode surface. (a) $30 \mathrm{~mm}$ length (i.e. $z_{1}=60 \mathrm{~mm}, z_{2}=90 \mathrm{~mm}$ ) with $V_{\mathrm{T}}=0.378 \times V_{0}$ and $z_{0}=150 \mathrm{~mm}$; (b) $45 \mathrm{~mm}$ (i.e. $z_{1}=90 \mathrm{~mm}, z_{2}=135 \mathrm{~mm}$ ) with $V_{\mathrm{T}}=0.282 \times V_{0}$ and $z_{0}=225 \mathrm{~mm}$. Plots are of a needle electrode of $1.5 \mathrm{~mm}$ diameter $\left(r_{i}=0.75 \mathrm{~mm}\right)$.

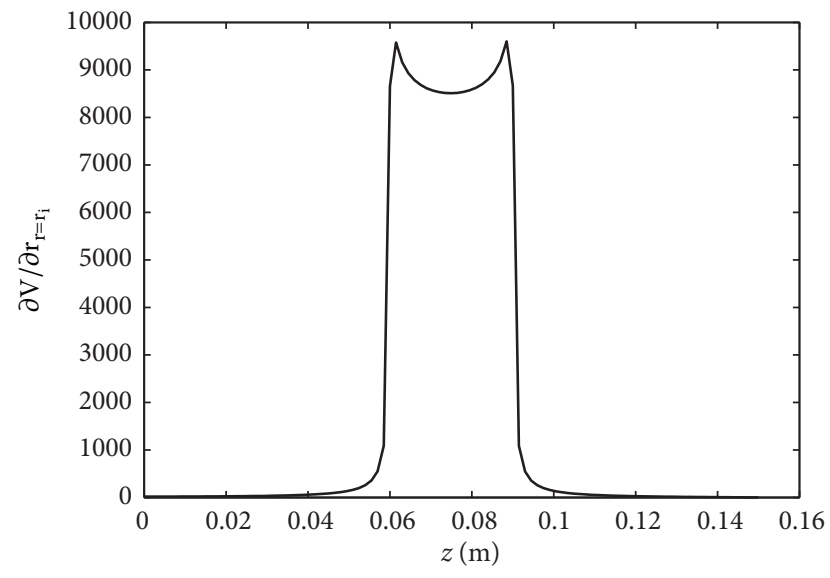

Figure 6: Profiles of $\partial V / \partial r$ computed at $r=r_{i}$. The value of $V_{T}$ was suitably changed to achieve a uniform voltage on the electrode surface. The plot is of a needle electrode with a conductive portion of $30 \mathrm{~mm}$. The following domain dimensions were considered: $r_{0}=$ $60 \mathrm{~mm}, z_{1}=60 \mathrm{~mm}, z_{2}=90 \mathrm{~mm}$, and $z_{0}=150 \mathrm{~mm}$.

Although most needle electrodes have a real physical structure based on hollow rather than solid bars, the lumen being used for instance to circulate cooled liquid, place thermocouples, introduces guide-wires, etc. In this respect, although our mathematical model did not consider the lumen, the electrical behavior is exclusively associated with the distribution of the electrical variables in the tissue, and hence what is included or not included in the electrode is irrelevant in terms of these electrical variables, as the electrode surface acts as a Faraday cage.

It is important to point out that the analytical solution was obtained by assuming uniform tissue surrounding the electrode. In particular, a constant value for electrical conductivity $\sigma$ had to be assumed in order to solve the electric problem using the Laplace Equation (Eq. (1)). Although the electrical conductivity changes significantly from tissue to tissue, it does not change much for a single type of well irrigated tissue, like the tissue around the electrode. The model herein presented is valid in situations where electrical conductivity is spatially and temporally constant, such as in electrostimulation. In the case of RF heating, the proposed solution is limited to represent the electric behavior (electric field, current density, and Joule heating) in an initial state, i.e., just before induced heating changes the $\sigma$ distributions.

For validation purposes, a comparison between this analytical solution and a numerical simulation using COMSOL 4.2a (COMSOL, Burlington, MA, USA), for the same dimensions and parameters, was conducted. The numerical solution, based on Finite Element Method, does not include the electrode as part of the computational domain, but includes the region $r<r_{\mathrm{i}}$ and $z>z_{2}$, not included in the analytical solution because the method of separation of variables requires the use of a regular geometry. The results obtained by both methods are shown in Figures 9 and 10 . Figures 9(a) and 9(b) compare vis-a-vis the voltage contour plots obtained by both methods. Figure 10 compares the voltage profiles along $z$ at a line where $r=0.75 \mathrm{~mm}$ (interface 


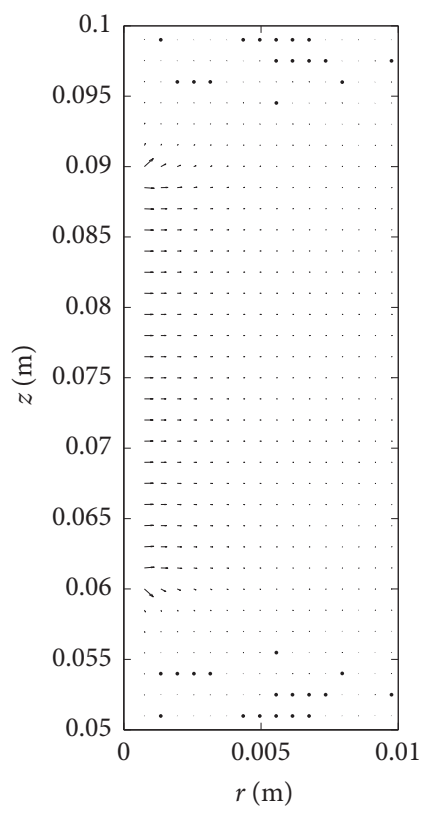

(a)

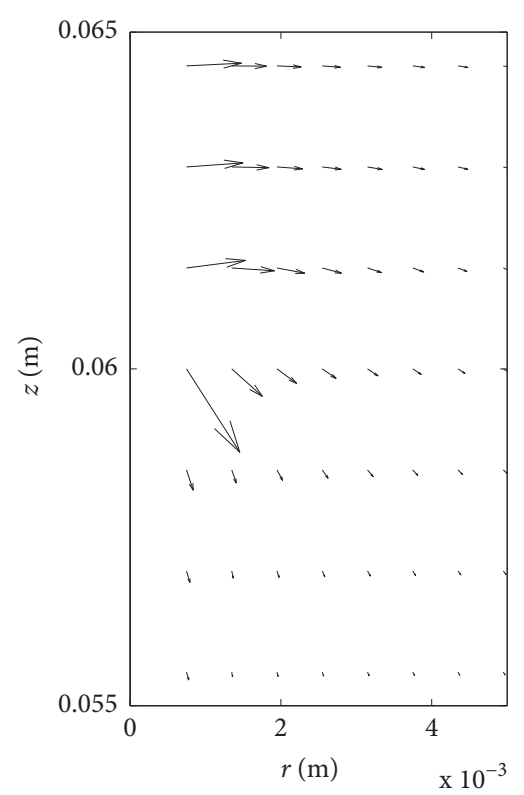

(b)

Figure 7: (a) Example of distribution of current density $\mathbf{J}$ in the vicinity of the electrode of a needle electrode with a conductive portion of $30 \mathrm{~mm}$. The following domain dimensions were considered: $r_{0}=60 \mathrm{~mm}, z_{1}=60 \mathrm{~mm}, z_{2}=90 \mathrm{~mm}$, and $z_{0}=150 \mathrm{~mm}$. (b) Detail of the distribution around the tip.

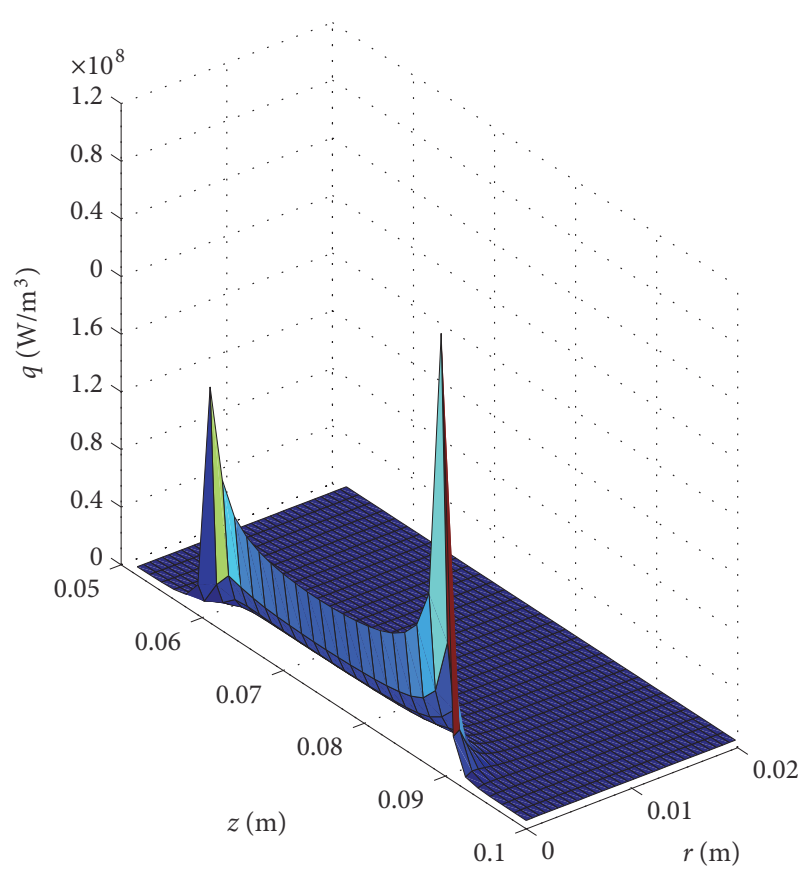

FIGURE 8: Example of distribution of Joule heating (q) in tissue of a needle electrode with a conductive portion of $30 \mathrm{~mm}$. The following domain dimensions were considered: $r_{0}=60 \mathrm{~mm}, z_{1}=60 \mathrm{~mm}, z_{2}=$ $90 \mathrm{~mm}$, and $z_{0}=150 \mathrm{~mm}$.

electrode-tissue). Both figures show the very good agreement in results obtained by both methods.

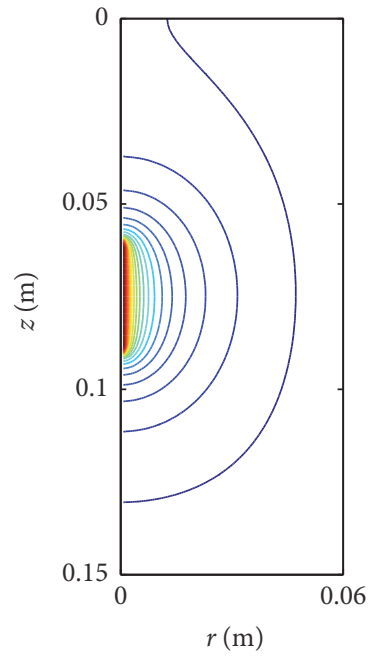

(a)

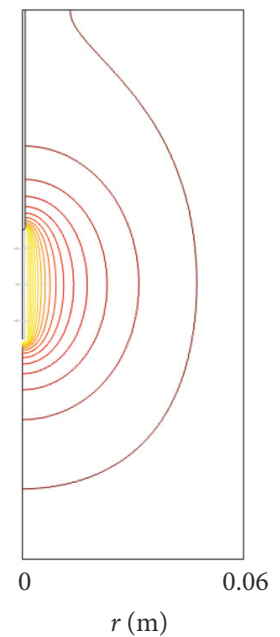

(b)
FIGURE 9: Comparison of voltage contours obtained by two methods: (a) analytical solution with $V_{\mathrm{T}}=0.378 V_{0}$ and (b) numerical solution using COMSOL. Contour lines for both cases are for values of voltage ranging from $0.625 \mathrm{~V}$ to $24.375 \mathrm{~V}$ at intervals of $1.25 \mathrm{~V}$.

\section{Conclusions}

A novel analytical solution is proposed, which allows accurate reproduction of the electric behavior of a needle electrode of given length surrounded by a homogenous space. Importantly, the solution also allows to reproduce the edge effect 


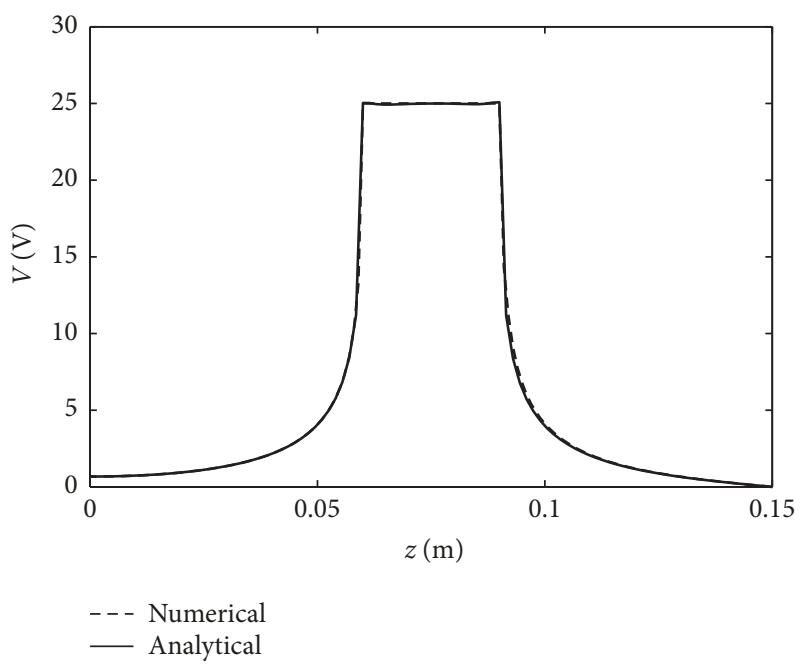

FIGURE 10: Comparison of voltage profiles along $\mathrm{z}$ at a line where $\mathrm{r}=0.75 \mathrm{~mm}$ obtained by two methods. Solid line for analytical solution with $V_{\mathrm{T}}=0.378 V_{0}$, dashed line for numerical solution using COMSOL.

characterized by an extremely high gradient around the electrode tip. The analytic solution was finally validated against a numerical solution based on a commercial software based on Finite Element Method.

\section{Data Availability}

The datasets generated during the current study are available from the corresponding author on reasonable request.

\section{Conflicts of Interest}

The authors declare that they have no conflicts of interest.

\section{Acknowledgments}

This work was supported by the Universidad Autónoma de San Luis Potosí (México), which granted R. Romero-Méndez who is on a sabbatical leave to do research in the field of biomedical engineering. This work was supported by the Spanish Ministerio de Ciencia, Innovación y Universidades under "Programa Estatal de I+D+i Orientada a los Retos de la Sociedad” (grant number: RTI2018-094357-B-C21).

\section{References}

[1] S. Mulier, Y. Miao, P. Mulier et al., "Electrodes and multiple electrode systems for radiofrequency ablation: a proposal for updated terminology," European Radiology, vol. 15, no. 4, pp. 798-808, 2005.

[2] D. R. Merrill, M. Bikson, and J. G. R. Jefferys, "Electrical stimulation of excitable tissue: design of efficacious and safe protocols," Journal of Neuroscience Methods, vol. 141, no. 2, pp. 171-198, 2005.

[3] S. F. Cogan, "Neural stimulation and recording electrodes," Annual Review of Biomedical Engineering, vol. 10, pp. 275-309, 2008.
[4] H. Kwon, S. B. Rutkove, and B. Sanchez, "Recording characteristics of electrical impedance myography needle electrodes," Physiological Measurement, vol. 38, no. 9, pp. 1748-1765, 2017.

[5] F. Burdío, E. J. Berjano, A. Navarro et al., "RF tumor ablation with internally cooled electrodes and saline infusion: what is the optimal location of the saline infusion?" Biomedical Engineering Online, vol. 6, article 30, 2007.

[6] B. Zhang, M. A. J. Moser, E. M. Zhang, Y. Luo, C. Liu, and W. Zhang, "A review of radiofrequency ablation: large target tissue necrosis and mathematical modelling," Physica Medica, vol. 32, no. 8, pp. 961-971, 2016.

[7] A. M. Samoudi, S. Kampusch, E. Tanghe et al., "Numerical modeling of percutaneous auricular vagus nerve stimulation: a realistic 3D model to evaluate sensitivity of neural activation to electrode position," Medical \& Biological Engineering \& Computing, vol. 55, no. 10, pp. 1763-1772, 2017.

[8] A. M. Samoudi, G. Vermeeren, E. Tanghe, R. Van Holen, L. Martens, and W. Josephs, "Numerically simulated exposure of children and adults to pulsed gradient fields in MRI," Journal of Magnetic Resonance Imaging, vol. 44, no. 5, pp. 1360-1367, 2016.

[9] M. Trujillo, J. Bon, M. José Rivera, F. Burdío, and E. Berjano, "Computer modelling of an impedance-controlled pulsing protocol for RF tumour ablation with a cooled electrode," International Journal of Hyperthermia, vol. 32, no. 8, pp. 931939, 2016.

[10] E. Ewertowska, B. Mercadal, V. Muñoz et al., "Effect of applied voltage, duration and repetition frequency of RF pulses for pain relief on temperature spikes and electrical field: a computer modelling study," International Journal of Hyperthermia, vol. 34, pp. 112-121, 2018.

[11] B. Zhang, M. A. J. Moser, E. M. Zhang, Y. Luo, and W. Zhang, "A new approach to feedback control of radiofrequency ablation systems for large coagulation zones," International Journal of Hyperthermia, vol. 33, no. 4, pp. 367-377, 2017.

[12] D. Haemmerich, L. Chachati, A. S. Wright, D. M. Mahvi, F. T. Lee Jr., and J. G. Webster, "Hepatic radiofrequency ablation with internally cooled probes: effect of coolant temperature on lesion size," IEEE Transactions on Biomedical Engineering, vol. 50, no. 4, pp. 493-500, 2003.

[13] J. A. López Molina, M. J. Rivera, and E. Berjano, "Analytical transient-time solution for temperature in non perfused tissue during radiofrequency ablation," Applied Mathematical Modelling: Simulation and Computation for Engineering and Environmental Systems, vol. 42, pp. 618-635, 2017.

[14] R. Romero-Méndez and E. Berjano, "An analytical solution for radiofrequency ablation with a cooled cylindrical electrode," Mathematical Problems in Engineering, vol. 2017, Article ID 9021616, 12 pages, 2017.

[15] E. Berjano, "Theoretical modeling for radiofrequency ablation: state-of-the-art and challenges for the future," Biomedical Engineering Online, vol. 5, pp. 1-17, 2006. 


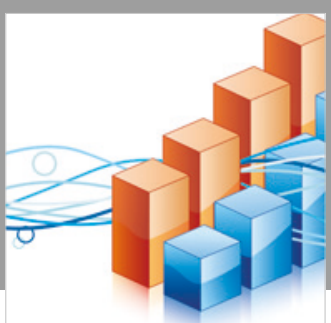

Advances in

Operations Research

\section{-n-m}
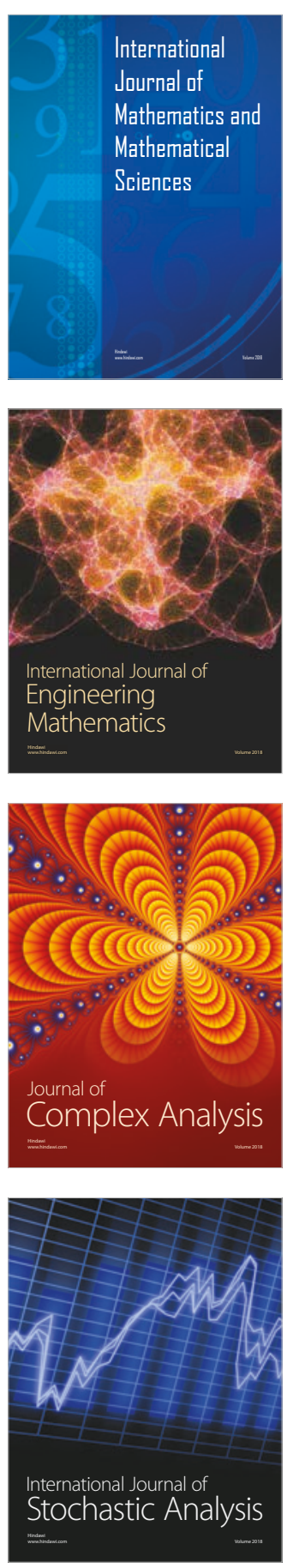
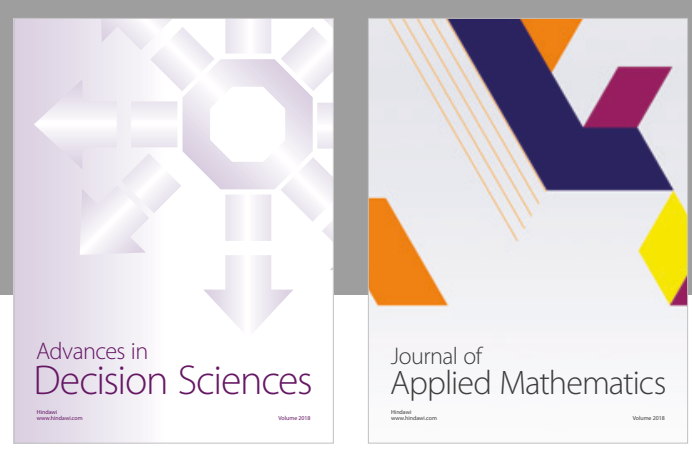

Journal of

Applied Mathematics
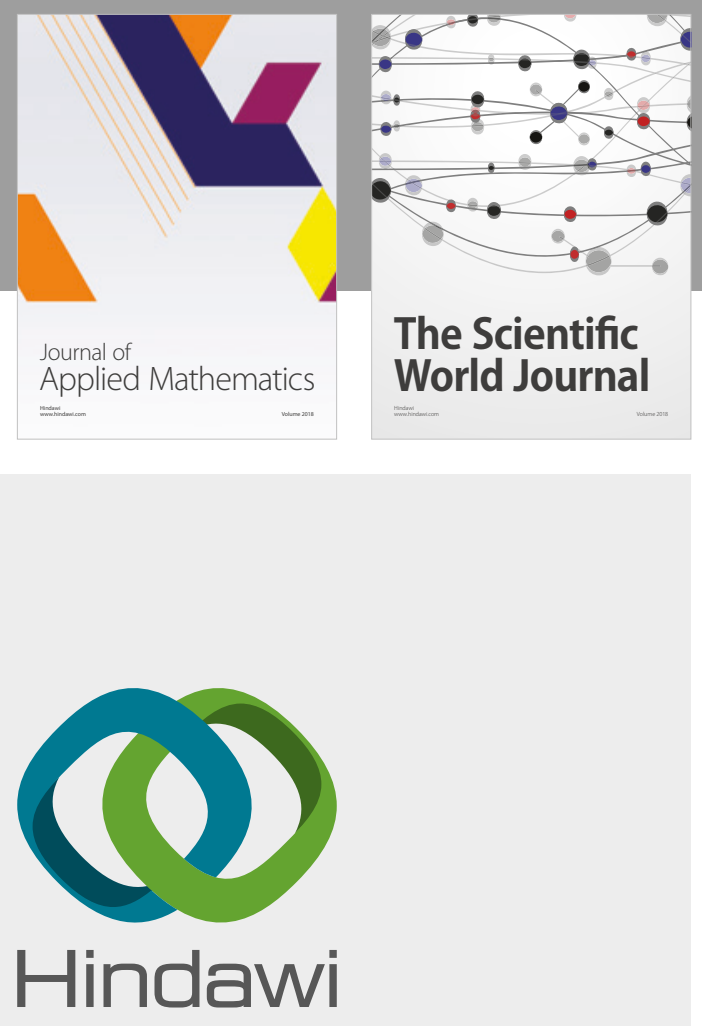

Submit your manuscripts at

www.hindawi.com

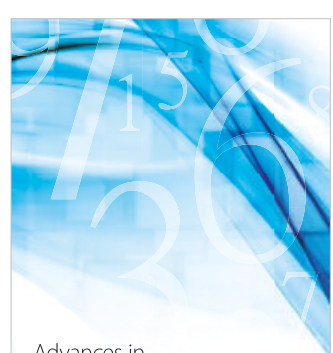

Advances in
Numerical Analysis
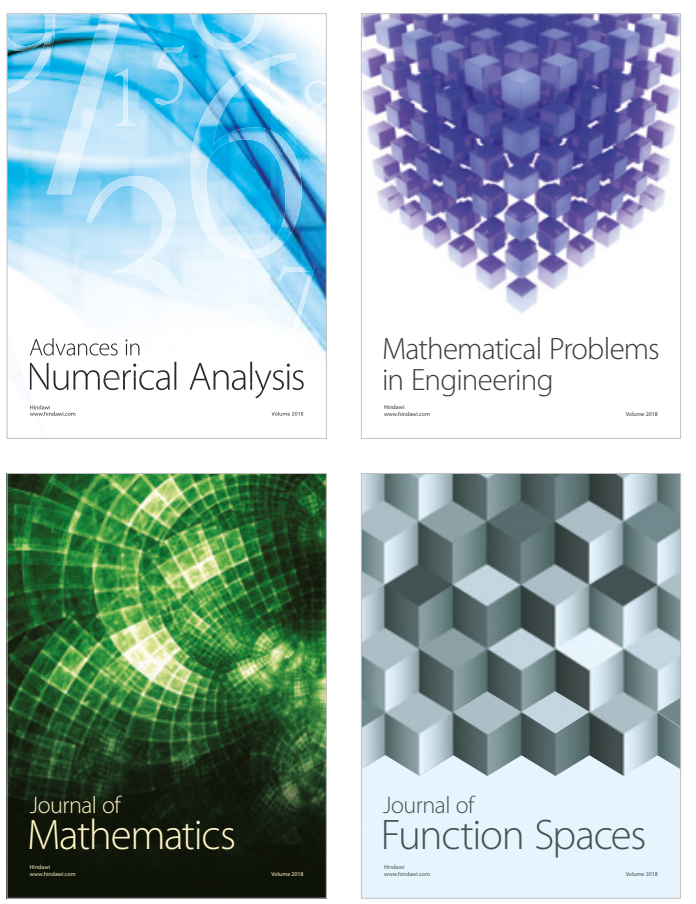

Mathematical Problems in Engineering

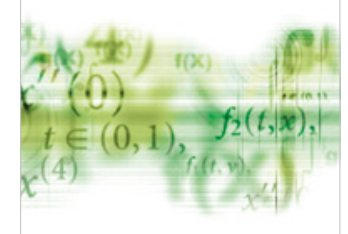

International Journal of

Differential Equations

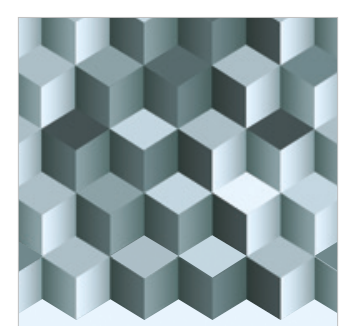

Journal of

Function Spaces

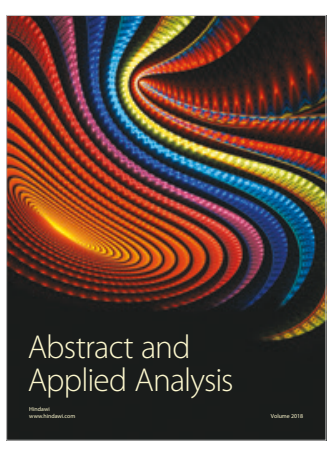

The Scientific

World Journal

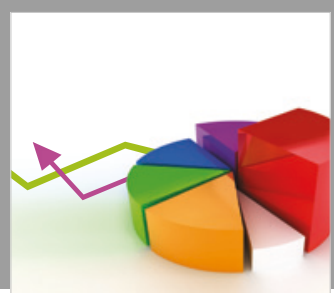

Journal of

Probability and Statistics
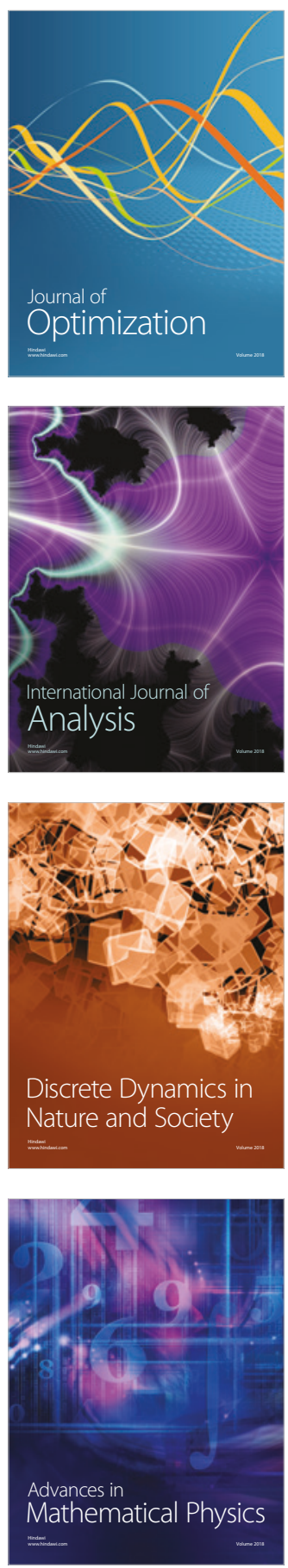